\title{
Endoscopic Third Ventriculostomy for an Idiopathic Disproportionately Large Communicating Fourth Ventricle: A Case Report
}

\author{
Hong-Gyu Yoon, Yong Ko, Young-Soo Kim, Koang-Hum Bak, Hyeong-Joong Yi, \\ Hyoung-Joon Chun, Kyu-Sun Choi, Shin-Woong Ko, Min-Kyun Na
}

Department of Neurosurgery, College of Medicine, Hanyang University, Seoul, Korea

\begin{abstract}
A disproportionately large communicating fourth ventricle (DLCFV) is a rare condition. A 34-year-old man experienced severe headaches, nausea, vomiting, and gait disturbance. Initial brain computed tomography (CT) showed markedly dilated ventricles with prepontine cistern shrinkage. Following extraventricular drain (EVD) insertion, approximately $400 \mathrm{~mL} / \mathrm{d}$ of cerebrospinal fluid (CSF) was removed over 8 days. During this time, no significant changes in ventricle size were observed on CT images or the facial pain scale (FPS). We then performed an endoscopic third ventriculostomy and changed the drainage catheter. Immediately after surgery, the patient's symptoms were relieved, and the drainage volume gradually decreased. EVD was successfully removed on the eighth postoperative day. Our experiences suggest that neurosurgeons should consider the importance of a third ventriculostomy for the diagnosis and treatment of DLCFV.
\end{abstract}

Key Words: Communicating hydrocephalus; Endoscopy; Ventriculostomy; Cerebrospinal fluid

\section{INTRODUCTION}

Disproportionately large communicating fourth ventricle (DLCFV) is a very rare condition with no clear pathophysiology [1]. DLCFV is often confused with an isolated fourth ventri-

Received: August 3, 2021 Revised: August 27, 2021

Accepted: August 28, 2021

Corresponding Author: Min-Kyun Na

Department of Neurosurgery, College of Medicine, Hanyang University,

222-1 Wangsimni-ro, Seongdong-gu, Seoul 04763, Korea.

Tel: +82-2-2290-8500, Fax: +82-2-2291-8498,

E-mail:mavmav@hanmail.net

ORCID: https://orcid.org/0000-0001-6826-8490 cle (IFV), also known as a trapped or encysted fourth ventricle. IFV is defined as a markedly dilated fourth ventricle due to obstruction of the aqueduct of Sylvius and foramina of Magendie and Luschka [2], whereas DLCFV features a dilated fourth ventricle but a patent aqueduct of Sylvius with no definite obstruction [3].

This report presents the case of a 34-year-old man with idiopathic DLCFV and no significant medical history who was successfully treated with an endoscopic third ventriculostomy (ETV).

\section{CASE REPORT}

A 34-year-old man with no clinical history visited our insti-

(C) 2021 Korean Neuro-Pain Society

(c) This is an Open Access article distributed under the terms of the Creative Commons Attribution Non-Commercial License (http://creativecommons.org/licenses/by-nc/4.0/) which permits unrestricted non-commercial use, distribution, and reproduction in any medium, provided the original work is properly cited. 
tution with severe headache, nausea, vomiting, and mild gait disturbance. The symptoms suddenly started 1 week before his visit and were refractory to pain killers. Laboratory tests revealed no significant abnormalities. Electrocardiography performed at admission revealed bradycardia with a first-degree atrioventricular (AV) block; however, transthoracic echocardiography showed no abnormalities or cardiac hypoperfusion.

Initial brain computed tomography (CT) showed markedly dilated third, fourth, and bilateral lateral ventricles with prepontine cistern shrinkage and severe brain parenchymal edema (Fig. 1A). Extraventricular drainage (EVD) was performed during the initial treatment. The intraoperative ventricular opening pressure was $30 \mathrm{cmH}_{2} \mathrm{O}$. Since the opening pressure was high, we assumed that the hydrocephalus was obstructive with a fourth ventricular outlet obstruction. However, postoperative enhanced brain CT showed no definite obstructive lesion in or near the fourth ventricle.

Postoperative intensive care unit treatment showed persistently high intracranial pressure (ICP) and symptoms despite an EVD volume exceeding $400 \mathrm{~mL}$ daily. Brain and upper cervical enhanced magnetic resonance imaging (MRI) studies for the further evaluation of obstructive lesions such as tumorous conditions failed due to poor patient participation. Despite the daily drainage of $>400 \mathrm{~mL}$ through the ventricular catheter, the patient's headache did not improve. Brain CT follow-up performed 8 days after the EVD insertion showed no remarkable difference in ventricular size or brain edema (Fig. 1B).

Therefore, we presumed a potential diagnosis of DLCFV and performed ETV under general anesthesia. In addition to ETV with an EVD catheter change, brain and cervical spine MRI enhancement studies were performed under general anesthesia. We used the previous EVD trajectory consisting of a right side burr hole site $1 \mathrm{~cm}$ anterior to the coronal suture and $3 \mathrm{~cm}$ lateral to the midline. We used a peel-away sheath, which enabled easy insertion of the endoscope into the ventricle without repeated trauma to the cerebral cortex. The right foramen of Monro was identified by confluence of the anterior septal vein, thalamostriate vein, and choroid plexuses (Fig. 2A). Before fenestration of the third ventricle floor, we identified the puncture site between the mammillary bodies and infundibular recess at the most transparent site (Fig. 2B) as well as a patent aqueduct of Sylvius (Fig. 2C). The third ventricle floor was bluntly perforated using flexible grasping forceps to reduce the risk of vascular injury (Fig. 2D, E). Finally, no signs of adhesion were found at the interpeduncular cistern (Fig. 2F). Cervical spine MRI showed prominent epidural vein engorgement, while brain MRI showed no tumorous conditions or infectious signs asso- ciated with the DLCFV (Fig. 3).

As the ICP began to decrease, the volume of the extraventricular drainage also started to decline. Brain CT performed 14 days after ETV showed a decreased fourth ventricle size and widened prepontine cistern (Fig. 1C), which led to decreased symptoms. Mild diplopia and cerebrospinal fluid (CSF) leakage
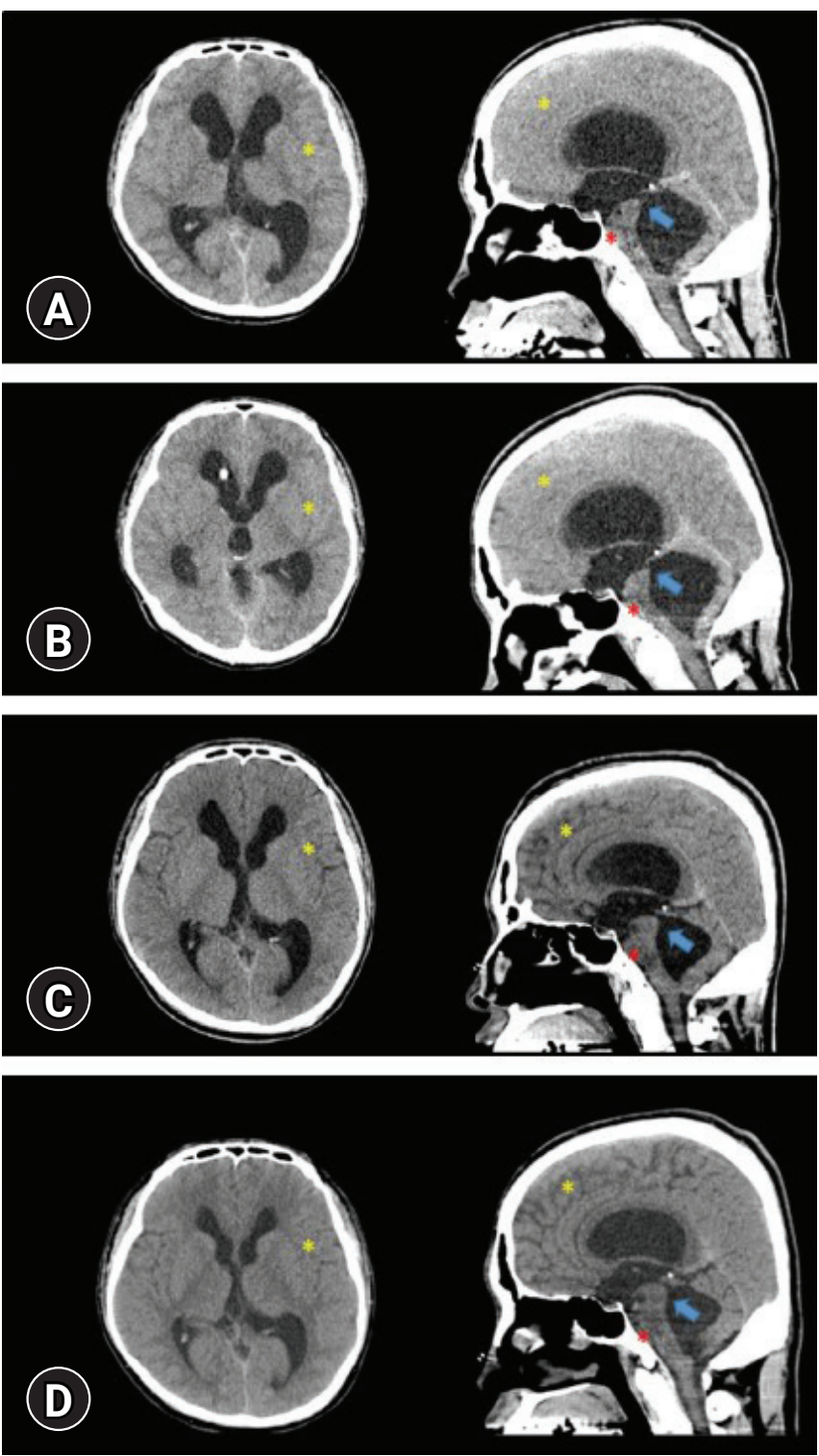

Fig. 1. Serial preoperative and postoperative computed tomography (CT) scans. (A) Preoperative, (B) 8 days after the external ventricular drainage (EVD) procedure, (C) 14 days after the third ventriculostomy procedure, and (D) 2 months after the third ventriculostomy procedure. The yellow asterisks indicate the gradual decreased parenchymal edema. The red asterisks show the enlarged prepontine cistern after the third ventriculostomy. The blue arrows indicate the decreased size of the fourth ventricle with relieved acute angle of the posterior surface of the midbrain. 

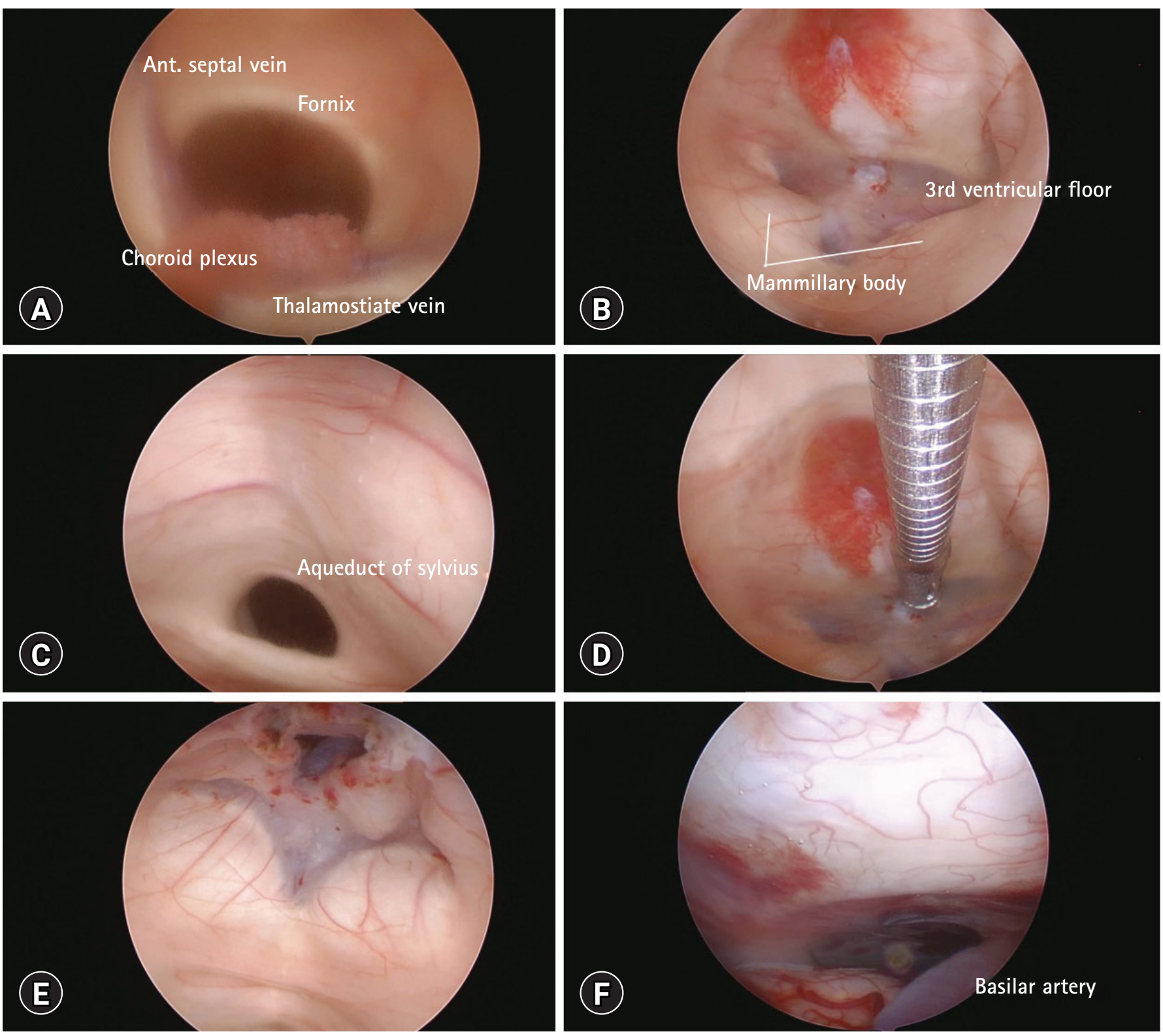

Fig. 2. Stages of endoscopic third ventriculostomy. (A) Right lateral ventricle view of the foramen of Monro. (B) Third ventricle floor with the infundibulo-mammillary triangle. (C) Patent dilated aqueduct of Sylvius. (D, E) Third ventriculostomy using flexible grasping forceps. (F) Interpeduncular cistern without adhesion.

from the surgical wound were observed postoperatively. However, the diplopia improved with conservative treatment, while the CSF leakage resolved with primary wound revision. The patient was discharged 36 days after admission. Brain CT performed 2 months later at the outpatient clinic showed a gradual reduction in ventricle size, including the fourth ventricle, a widened prepontine cistern, and relieved parenchymal edema (Fig. 1D). The patient's headache resolved with no other complications.

\section{DISCUSSION}

DLCFV is a rare disease characterized by dilatation of the fourth ventricle regardless of lateral ventricle size without definite obstruction of the cerebral aqueduct, indicating patency of the inlet of the fourth ventricle [3]. Its treatment has not been thoroughly established owing to the characteristic hemodynamics of the CSF and a poor understanding of its etiology and pathophysiology [2,4]. Treatment for obstructive hydrocephalus includes ventricular drainage, ventriculoperitoneal shunting 
(VP), lowering of the opening pressure of the adjustable valve, ETV, aqueductoplasty with or without stent placement, interventriculostomy, septostomy, foraminoplasty, and decompressive craniectomy of the posterior fossa [5-7].

Various hypotheses have been proposed to explain the pathophysiology of DLCVF. A previous study suggested that any obstructive lesion at the outlet of the fourth ventricle results in increased ventricular pressure due to CSF pulse waves and the water hammer effect. Therefore, EVD or VP shunting cannot be the ultimate treatment; rather, these operations could worsen the patient's condition due to supratentorial overdrainage and eventually increase the pressure gradient between the infratentorial and supratentorial cavities [8]. The patient in the present report initially underwent EVD. However, as his symptoms did not improve, his daily drainage exceeded $400 \mathrm{~mL}$ for 8 days, and follow-up brain CT showed no change in ventricu-

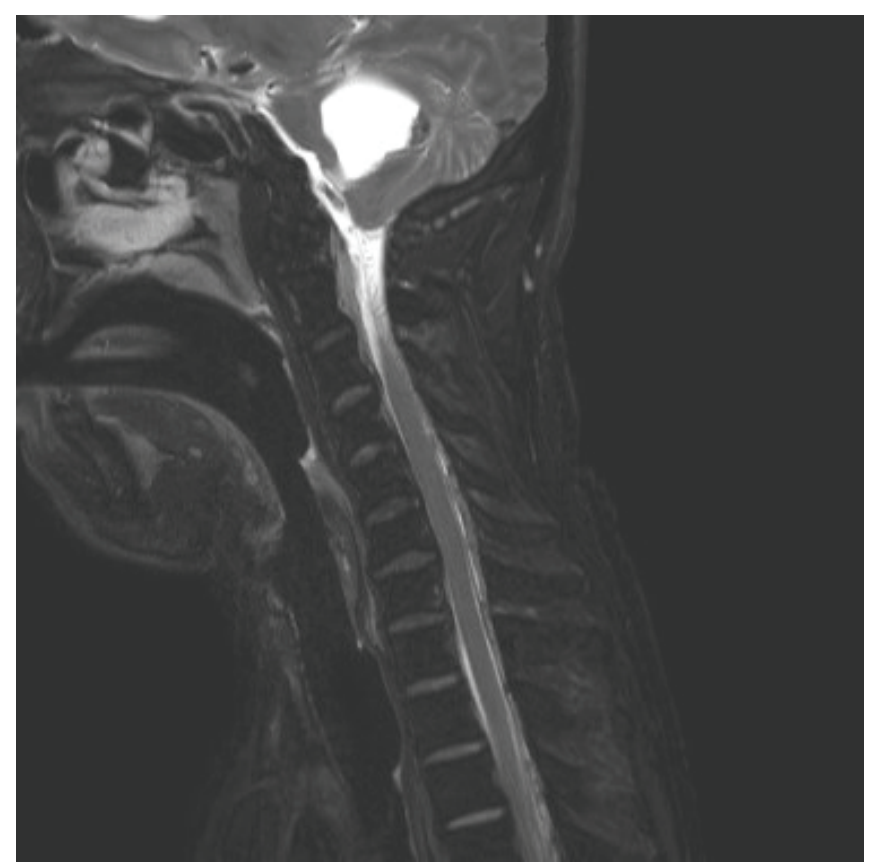

Fig. 3. Cervical magnetic resonance image showing prominent vein engorgement and no evidence of syringomyelia. lar size, ETV was performed.

Kawabata et al. [3] proposed the diagnosis of DLCFV using radiologic imaging of a dilated fourth ventricle and intraoperative endoscopic findings of a patent aqueduct of Sylvius and suggested ETV as a first-line treatment. In our case, based on this information, we conducted ETV after intraoperatively confirming the patency of the aqueduct of Sylvius. The patient's headache, nausea, and vomiting gradually improved soon thereafter (Table 1).

We conducted a PubMed search for original studies on idiopathic DLCFV treatment in patients without medical or surgical histories that could be related to hydrocephalus. The search revealed 14 patients who were diagnosed with DLCFV and underwent various successful treatments (Table 2). Lyu et al. [2] suggested that DLCFV treatment be individualized. As DLCFV is characterized by a patent aqueduct of Sylvius, it is reasonable to assume that most patients with DLCFV can benefit from supratentorial CSF shunt placement; however, not all are treated because of unresolved infratentorial- supratentorial pressure gradients. These patients require additional ETV or a direct fourth ventricular shunt due to functional obstruction of the patent aqueduct of Sylvius [9].

The patients in this study experienced prolonged symptoms. Serial CT follow-up showed an unchanged enlarged ventricle size after EVD. This finding supported the hypothesis of functional obstruction of the aqueduct of Sylvius with fourth ventricle outlet obstruction, which were resolved after ETV. However, we suggest that the focus be on CSF production and dynamics. The patient's symptoms were not correlated with the amount of CSF drainage from the EVD, which suggests that the amount of drainage was not determined by the pressure gradient of the infratentorial-supratentorial cavity.

Furthermore, the CSF drainage volume remained constant regardless of the height of the EVD drainage bag between 20 $\mathrm{cm}$ above and $5 \mathrm{~cm}$ below the tragus level. Therefore, for some patients with DLCFV, lowering the supratentorial pressure cannot fully treat DLCFV. The CSF produces $70 \%$ of the choroid

Table 1. Daily changes in variables during hospitalization

\begin{tabular}{lcccccccccccccccccc}
\hline HD (d) & 1 & 2 & 3 & 4 & 5 & 6 & 7 & 8 & 9 & 10 & 11 & 12 & 13 & 14 & 15 & 16 & 17 & 18 \\
POD_EVD (d) & 0 & 1 & 2 & 3 & 4 & 5 & 6 & 7 & 8 & 9 & 10 & 11 & 12 & 13 & 14 & 15 & 16 & 17 \\
POD_ETV (d) & & & & & & & & & & 0 & 1 & 2 & 3 & 4 & 5 & 6 & 7 & 8 \\
EVD height* $(\mathrm{cm})$ & 20 & 20 & 20 & 10 & 10 & 10 & 0 & -5 & -5 & 10 & 10 & 10 & 10 & 10 & 10 & 10 & 10 & 10 \\
EVD volume $(\mathrm{mL})$ & 92 & 370 & 438 & 406 & 422 & 421 & 448 & 463 & 416 & 388 & 480 & 398 & 400 & 387 & 136 & 0 (clamp) & 200 & 150 \\
FPS & 8 & 10 & 8 & 8 & 10 & 8 & 8 & 8 & 8 & 8 & 8 & 6 & 6 & 4 & 4 & 2 & 2 & 2 \\
\hline
\end{tabular}

HD: hospital day, POD: postoperative day, EVD: extraventricular drainage, ETV: endoscopic third ventriculostomy, FPS: facial pain scale. *EVD height was measured based on the tragus level. 
Table 2. Presenting treatment and outcomes of 15 patients with idiopathic DLCFV reported in the literature and the present study

\begin{tabular}{lcccll}
\hline Author (yr) & Age (yr), sex & Etiology & Operative history & Treatment & Outcome \\
\hline Zimmerman et al. (1978) [10] & $43, \mathrm{M}$ & Idiopathic & None & VP shunt & Improved \\
Rifkinsonmann et al. (1987) [11] & $42, \mathrm{M}$ & Idiopathic & None & Suboccipital craniectomy excision of the outlet membrane & Improved \\
& $52, \mathrm{M}$ & Idiopathic & None & Suboccipital craniectomy excision of the outlet membrane & Improved \\
Mohanty et al. (1999) [12] & $32, \mathrm{M}$ & Idiopathic & None & ETV & Improved \\
& $20, \mathrm{~F}$ & Idiopathic & None & ETV & Improved \\
& $45, \mathrm{~F}$ & Idiopathic & None & ETV & Improved \\
Huang et al. (2001) [13] & $15, \mathrm{~F}$ & Idiopathic & None & Suboccipital craniotomy excision of the outlet membrane & Improved \\
Karachi et al. (2003) [14] & $21, \mathrm{~F}$ & Idiopathic & None & ETV & Improved \\
& $53, \mathrm{~F}$ & Idiopathic & None & ETV & Improved \\
& $68, \mathrm{M}$ & Idiopathic & None & ETV & Improved \\
Longatti et al. (2006) [15] & $64, \mathrm{~F}$ & Idiopathic & None & Opening of the foramen of Magendie \\
Hagihara et al. (2007) [16] & $13, \mathrm{M}$ & Idiopathic & None & VP shunt \\
Kawabata et al. (2020) [3] & $67, \mathrm{~F}$ & Idiopathic & None & ETV & Improved \\
& $46, F$ & Idiopathic & None & ETV & Improved \\
Present study (2021) & $34, \mathrm{M}$ & Idiopathic & None & ETV
\end{tabular}

DLCFV: disproportionately large communicating fourth ventricle, VP: ventriculoperitoneal, ETV: endoscopic third ventriculostomy.

plexuses of the lateral, third, and fourth ventricles and is produced at a rate of $500-700 \mathrm{~mL} / \mathrm{d}$. CSF flow is caused by pulsating blood vessels, respiration, and postural changes. As our patient's symptoms were relieved after the ETV, he may have had an abnormal CSF flow.

The patient's symptoms were relieved as the lateral and fourth ventricle sizes decreased. ETV enables direct CSF flow from the third ventricle to the prepontine cistern, which eventually reaches the arachnoid villi. Fig. 1 shows the progressive change on CT scans and ultimately a new equilibrium state after the ETV. The collapsed prepontine cistern was filled with CSF from the third ventricle and the cerebellar gyri became visible with supracerebellar-infratentorial space widening. We assumed that the ETV decreased the volume loading of the fourth ventricle by turning partial CSF flow directly to the prepontine cistern, and the decreased size of the fourth ventricle itself contributed to opening of the outlet obstruction, which was caused by downward herniation of the compressed cerebellar cortex or tonsil by the elevated fourth ventricular pressure. ETV not only lowers the volume loading of the fourth ventricle, it increases the CSF outflow to the subarachnoid space. In a future study, we plan to perform cisternography to confirm this supposition.

DLCFV is a rare disease with a problematic treatment choice. ETV can confirm the patency of the aqueduct, which is helpful for both diagnosis and treatment. For patients with interpeduncular cisternal adhesion or unsatisfactory outcomes after ETV, fourth ventricle shunting or suboccipital decompression should be considered.

\section{CONFLICTS OF INTEREST}

No potential conflict of interest relevant to this article was reported.

\section{REFERENCES}

1. Yamashita T, Hiramatsu H, Kitahama Y, Tokuyama T, Sugiyama K, Namba H. Disproportionately large communicating fourth ventricle associated with syringomyelia and intradural arachnoid cyst in the spinal cord successfully treated with additional shunting. Case report. Neurol Med Chir (Tokyo) 2012;52:231-234

2. Lyu J, Wang JB, Quan Y, Gong S. Disproportionately large communicating fourth ventricle: two case reports. J Med Case Rep 2019;13:222

3. Kawabata T, Takeuchi K, Nagata Y, Ishikawa T, Choo J, Wakabayashi T. Endoscopic treatment strategy for a disproportionately large communicating fourth ventricle: case series and literature review. Neurol Med Chir (Tokyo) 2020;60:351359

4. Ferrer E, de Notaris M. Third ventriculostomy and fourth ventricle outlets obstruction. World Neurosurg 2013;79:S20; e9-13

5. Antes S, Salah M, Linsler S, Tschan CA, Breuskin D, Oertel J. Aqueductal stenting with an intra-catheter endoscope--a 
technical note. Childs Nerv Syst 2016;32:359-363

6. Sagan LM, Kojder I, Poncyljusz W. Endoscopic aqueductal stent placement for the treatment of a trapped fourth ventricle. J Neurosurg 2006;105:S275-S280

7. Sharifi G, Alavi E, Rezaee O, Jahanbakhshi A, Faramarzi F. Neuroendoscopic foraminoplasty for bilateral idiopathic occlusion of foramina of Monro. Turk Neurosurg 2012;22: 265-268

8. Ogiwara H, Morota N. Endoscopic transaqueductal or interventricular stent placement for the treatment of isolated fourth ventricle and pre-isolated fourth ventricle. Childs Nerv Syst 2013;29:1299-1303

9. Oi S, Matsumoto S. Pathophysiology of aqueductal obstruction in isolated IV ventricle after shunting. Childs Nerv Syst 1986;2:282-286

10. Zimmerman RA, Bilaniuk LT, Gallo E. Computed tomography of the trapped fourth ventricle. AJR Am J Roentgenol 1978;130:503-506

11. Rifkinson-Mann S, Sachdev VP, Huang YP. Congenital fourth ventricular midline outlet obstruction. Report of two cases. J Neurosurg 1987;67:595-599

12. Mohanty A, Anandh B, Kolluri VR, Praharaj SS. Neuroendoscopic third ventriculostomy in the management of fourth ventricular outlet obstruction. Minim Invasive Neurosurg 1999;42:18-21

13. Huang YC, Chang CN, Chuang HL, Scott RM. Membranous obstruction of the fourth ventricle outlet. A case report. Pediatr Neurosurg 2001;35:43-47

14. Karachi C, Le Guérinel C, Brugières P, Melon E, Decq P. Hydrocephalus due to idiopathic stenosis of the foramina of magendie and luschka. Report of three cases. J Neurosurg 2003;98:897-902

15. Longatti P, Fiorindi A, Feletti A, Baratto V. Endoscopic opening of the foramen of magendie using transaqueductal navigation for membrane obstruction of the fourth ventricle outlets. Technical note. J Neurosurg 2006;105:924-927

16. Hagihara N, Sakata S. Disproportionately large communicating fourth ventricle with syringomyelia: Case report. Neurol Med Chir (Tokyo) 2007;47:278-281 